\title{
Buffer analysis of the explicit rate congestion control mechanism for the ABR service category in ATM Networks
}

\author{
C. Blondia ${ }^{\dagger}$, O. Casals ${ }^{\ddagger}$, B . Van Houdt $t^{\dagger}$ \\ ${ }^{\dagger}$ University of Antwerp, Dept. Math. and Computer Science \\ Universiteitsplein, 1, B-2610 Antwerp - Belgium, \\ $\ddagger$ Polytechnic, University of Catalunya, Computer Architecture Dept., \\ C/ Gran Capitan, s/n Campus Norte, D6, E-08071 Barcelona - Spain, \\ \{blondia, vanhoudt $\}$ @uia.ua.ac.be, olgalac.upc.es
}

\begin{abstract}
In this paper we consider an $A B R$ traffic stream which shares an output port of a switch with delay sensitive CBR/VBR traffic. Congestion control of the ABR traffic is achieved by means of an Explicit Rate congestion control scheme. The occupancy of the ABR-buffer in the switch is analytically evaluated. Application of the analysis on numerical examples illustrates the influence of the following system characteristics on the buffer occupation. From this study some guidelines and engineering rules are derived for the ABR service category in ATM networks.
\end{abstract}

\section{Keywords}

ATM, Traffic Management, Congestion Control, Available Bit Rate, Explicit Rate Congestion Control

\section{INTRODUCTION}

In order to allow for different service types, each with their specific Quality of Service (QoS) requirements, the Asynchronous Transfer Mode (ATM) needs adequate traffic management mechanisms. For real-time service categories, such as CBR and rt-VBR traffic services, the network applies preventive open loop control mechanisms. CAC, UPC/NPC and Traffic Shaping belong to this class of traffic control mechanisms. Open loop control requires an adequate prediction and control of the traffic volume and its profile. This is achieved by means of a traffic contract established between the source and the network at call set-up. For data traffic services, such a prediction is difficult (if not impossible), and therefore open loop schemes are not efficient in this case, as 
they may result in a considerable waste of network resources. For this class of traffic services, a closed loop control scheme seems to be more appropriate since it may use the remaining bandwidth more efficiently. Such a closed loop scheme dynamically regulates the cell rate of a connection based on feedback information from the network. For the ABR service class, there are strict QoS guarantees towards the Cell Loss Ratio (CLR), but no guarantees towards delay or delay variation. The network and the source may agree upon a Minimum Cell Rate (MCR) and a Peak Cell Rate (PCR). Between MCR and PCR, the network guarantees a low CLR, as long as the Source End Station (SES) adapts its cell rate to the feedback information received from the network. Since the notification process involves a round trip delay of twice the distance between SES and switch, the network has to provide large buffers in the switches to cope with the low cell loss guarantees in the presence of this notification delay. Within the ATM Forum ([1]), a number of congestion control mechanisms for the ABR service class have been proposed, which differ in the way the feedback is realized. The Binary Feedback Congestion Control mechanism and the Explicit Rate Congestion Control scheme are the most important ones. Although the ATM Forum traffic management specifications allow the older switches with binary feedback, the newer explicit rate switches will provide better performance and faster control. In this last class of schemes, switches compute the rate a source should use to emit cells, called Explicit Rate (ER), and this rate is communicated to the source by means of Resource Management (RM) cells. From this ER, the source determines the Allowed Cell Rate (ACR) according to an algorithm specified by the ATM Forum (see [1]). This rate always satisfies the relationship $M C R \leq A C R \leq P C R$. Several ways of computing the ER have been proposed; e.g. Enhanced Proportional Rate Control Algorithm (EPRCA), Explicit Rate Indication for Congestion Avoidance (ERICA) and the new version named "ERICA+", the Congestion Avoidance using Proportional Control (CAPC), etc... (see [7, 8, 9, 2] ).

Several authors have proposed analytical models to derive the throughput and buffer requirements for ABR traffic when switches with binary feedback are used (see $[5,6,12,13,14,18]$ ). In this paper, we evaluate the required buffer space to guarantee a low cell loss (e.g. $<10^{-9}$ ) for the ABR traffic (see Figure 1). The switch used is based on the ERICA scheme ([8])), where the Allowed Cell Rate is updated on a periodical basis (every $P$ timeslots). We consider two traffic sources, a CBR/VBR traffic source and an ABR traffic source, both connected to a switch. The CBR/VBR traffic source generates a variable bit rate traffic, modeled by means of a D-BMAP (see [3, 4]), while for the ABR traffic source two cases are considered, namely a greedy (or persistent) ABR traffic source and an on/off ABR traffic source. The model takes the distance between ABR-SES and switch ( $2 \tau$ timeslots) into account. We derive the queue length distribution of the ABR queue in the switch. Two different cases are considered, each involving a different approach for the analysis : $2 \tau \leq P$ and $2 \tau>P$. In numerical examples, we investigate the influence 
of the following system characteristics on this performance measure : (i) the distance between the ABR source and the switch, (ii) the variability of the CBR/VBR traffic, (iii) the frequency by which the Allowed Cell Rate of the ABR source is updated, (iv) the burstiness of the ABR traffic.

\section{THE QUEUEING MODEL}

\subsection{System Configuration}

We evaluate the ABR buffer occupation in a system consisting of two end stations and a switch, a CBR/VBR SES and an ABR SES. Both the ABR and CBR/VBR traffic are input to the switch and are competing for the bandwidth of the same output port in the switch. The switch acts as a virtual destination station for the ABR traffic. The distance between the ABR-SES and the switch is $\tau$ time slots, where a time slot is the time needed to process a cell in the switch (and chosen as time unit).

\subsection{Source and Switch Behavior}

\section{a. $C B R / V B R$ Traffic Model}

The CBR/VBR traffic is modeled by means of a discrete-time Batch Markovian Arrival Processes (D-BMAP), a generic traffic model for VBR traffic in ATM networks (see [3],[4]). In particular, the superposition of on/off processes, a model for VBR video traffic, belongs to this class.

Consider $M$ discrete-time on/off sources, with geometrically distributed on period (mean on period $p$ time slots), geometrically distributed off period (mean off period $q$ time slots) and a probability of $1 / d$ to generate a cell in a slot during the on period. The superposition of these on/off sources can be modeled as a D-BMAP with matrices $\mathbf{D}_{n}, n \geq 0$, where the matrix $\mathbf{D}_{0}$ with elements $\left(d_{0}\right)_{i, j}$ governs transitions without arrivals, while the matrices $\mathbf{D}_{n}$ with elements $\left(d_{n}\right)_{i, j}, 1 \leq n \leq M$, govern transitions that correspond to arrivals of batches of size $n$.

\section{b. ABR Traffic Model}

We consider two cases : case A where the ABR source is a persistent (or greedy) source, i.e. this source has always cells to transmit and will do so at maximal allowed cell rate, and case $B$ where the ABR source is an on/off source. This on/off traffic is characterized by three parameters : the mean on period, the mean off period and the cell rate while in an on period. The rate at which cells can be transmitted is called the Allowed Cell Rate (ACR). The ACR varies between the Peak Cell Rate (PCR) and the Minimum Cell Rate 
(MCR), both values being defined at call setup. Every $P$ time slots, the ABRSES receives a notification of the new ACR to be used during the next $P$ time slots from the switch by means of the value in the ER field in a backward RM cell. The interval $P$ is called an observation period. It determines the time scale according to which the ACR adapts to the state of the network. We simplify the behavior of the SES as we let the ACR be completely determined by the ER, not taking into account additive increase and multiplicative decrease factors (see [1]). In case A, the ABR-SES generates traffic according to the allowed rate ACR, while in case $B$, the actual rate that is used depends on both the allowed rate ACR and the state of the ABR source.

\section{c. Switch Behavior}

The behavior of the switch shows many similarities to the ERICA switch described in [7]. During an observation period of $P$ time slots, the switch counts the number of arrivals from both the CBR/VBR traffic source and from the ABR traffic source. Denote these numbers by $N_{c}$, resp. $N_{a}$. The total input rate of the switch during this observation period is then $i=$ $\left(N_{c}+N_{a}\right) / P$ cells per slot. Let the desired utilization of the output link of the switch be TCR (Target Cell Rate). Then the overload factor is given by $o=i / T C R$. The explicit rate communicated to the SES is given by $r=$ $\min \left[P C R, \quad \max \left[M C R, \frac{N_{a} / P}{o}\right]\right] .$. We let the range of possible rate values be discrete and chose the value closest to $r$. We assume that ABR traffic is guaranteed a service rate of at least MCR.

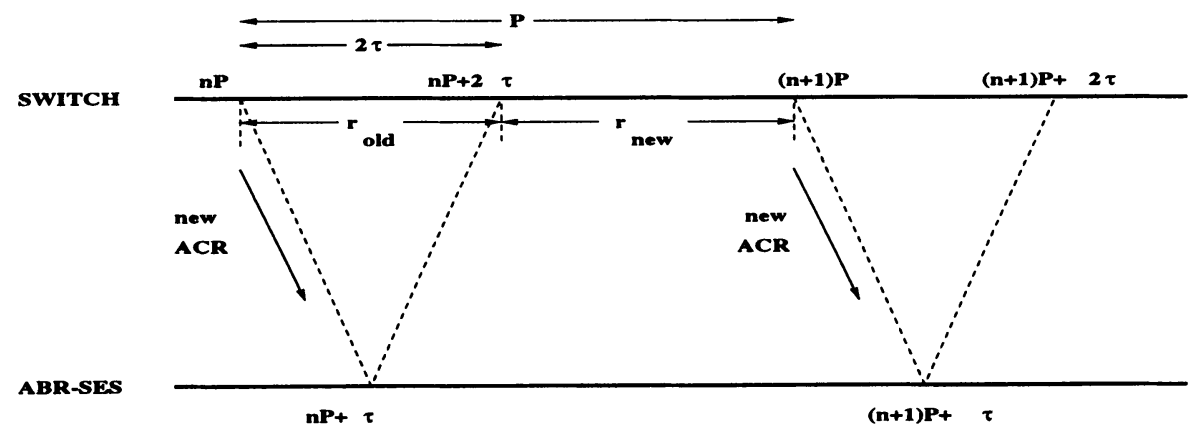

Figure 1 The interaction between Switch and ABR-SES, $2 \tau \leq P$

\subsection{Buffer Occupation}

The performance measure to be evaluated is the occupation of the ABR buffer in the switch. In particular we are interested in the $10^{-9}$-quantile of the queue length distribution of the ABR traffic. 


\section{ANALYSIS OF THE QUEUEING MODEL}

\subsection{The Buffer Occupation in case $2 \tau \leq P$}

\section{(a) Evolution of the Process}

Figure 2 illustrates the interaction between the ABR-SES and the switch in case $2 \tau \leq P$. Observe that during an observation period of length $P$, two rates for the ABR traffic apply. Indeed, consider the interval $[n P,(n+1) P[$ : Denote the rate during the interval $\left[(n-1) P+2 \tau, n P+2 \tau\left[\right.\right.$ by $r_{\text {old }}(n)$ and $r_{n e w}(n)$ the rate during the subinterval $[n P+2 \tau,(n+1) P[$. Let us describe the system at the end of each observation period, i.e. at instances $n P, n=1,2,3, \ldots$, by means of the following vector $\bar{S}\left(p_{c}(n), p_{a}(n), r_{\text {old }}(n), r_{n e w}(n)\right)=\bar{S}(n)$, with

- $p_{c}(n)$ is the phase of the CBR/VBR traffic at instant $n P$;

- $p_{a}(n)$ is the state of the ABR traffic source at instant $n P-\tau$;

- $r_{\text {old }}(n)$ is the ACR computed by the switch at the end of the observation period $[(n-2) P,(n-1) P[$

- $r_{\text {new }}(n)$ is the ACR computed by the switch at the end of the observation period $[(n-1) P, n P[$;

We compute the transition $\bar{S}(n) \longrightarrow \bar{S}(n+1)$. Let $\bar{S}(n)=\left(i_{0}, j_{0}, \mu_{0}, \nu_{0}\right)$.

(i) First we compute the joint distribution of the number of CBR/VBR arrivals and the phase of this process. Denote $N_{c}\left[t_{0}, t_{1}\right]$ the number of CBR/VBR arrivals during the interval $\left[t_{0}, t_{1}[\right.$. Then it is possible to compute this probability directly from the matrices $\mathbf{D}_{n}$, as $\mathbf{P}\left\{p_{c}(n+1)=i_{1}, N_{c}[n P,(n+1) P]=l \mid\right.$ $\left.p_{c}(n)=i_{0}\right\}$.

(ii) (this step is only necessary in case B) Compute the joint distribution of the number of $\mathrm{ABR}$ arrivals and the state of the ABR source during an interval of length $P: \mathbf{P}\left\{p_{a}(n+1)=j_{1}, N_{a}[n P,(n+1) P]=k \mid p_{a}(n)=j_{0}\right\}$. (iii) Clearly $r_{\text {old }}(n+1)=r_{\text {new }}(n)$.

(iv) Now we compute $r_{\text {new }}(n+1)$. We need to compute the number of ABR arrivals during $[n P,(n+1) P[$. First we consider case A. As two ACRs apply, we compute the two corresponding components separately. During $[n P, n P+2 \tau[$ the rate $r_{\text {old }}(n)=\mu_{0}$ applies, hence the number of ABR arrivals during that period equals $2 \tau \mu_{0}$. Similarly, the number of ABR arrivals during the interval $\left[n P+2 \tau,(n+1) P\left[\right.\right.$ is given by $(P-2 \tau) \nu_{0}$. Hence, the total number of ABR arrivals is $N_{a}[n P,(n+1) P]=\left\lfloor 2 \tau \mu_{0}+(P-2 \tau) \nu_{0}\right\rfloor$. In case B, the number of $\mathrm{ABR}$ arrivals is given by the computation in (ii). If there are $N_{c}[n P,(n+1) P]$ CBR/VBR arrivals (see (i)), then the new ACR is given by $r_{\text {new }}(n+1)=\min \left[P C R, \quad \max \left[M C R, \frac{r_{\text {new }}(n)}{N_{\text {tot }} / P} T C R\right]\right]$, with $N_{\text {tot }}=N_{c}[n P,(n+1) P]+N_{a}[n P,(n+1) P]$. 


\section{(b) Buffer Analysis}

From the above analysis, we know the number of ABR cells that have arrived at the $\mathrm{ABR}$ queue in the switch during an interval $[n P,(n+1) P[$. Let this number be denoted by $N_{a}$. The CBR/VBR traffic has priority, i.e. is served first, but such that ABR traffic has a guaranteed service rate of MCR, i.e. of every $P$ slots there are $M C R \times P$ slots reserved for ABR cells (if there are ABR cells available). Hence, if $N_{c}$ cells of CBR/VBR traffic arrive during $[n P,(n+1) P[$, and taking into account Remark 1 , the number of slots available for serving ABR cells, is given by $B=\max \left[M C R \times P, P-N_{c}\right]$. Denote by $Q(n)$ the queue length of the ABR buffer in the switch at instant $n P$. Then we can describe approximately the evolution of the queue length as follows: $Q(n+1)=\max \left[0, \quad Q(n)+N_{a}-B\right]$. The process $Q(n)$ forms a Markov chain with the following transition matrix. Let $C_{i}$ the matrix describing the transition of the variables $\left(p_{c}, p_{a}, r_{\text {old }}, r_{\text {new }}\right)$ giving rise to a growth $i$ in number of ABR cells during an observation period $P$. Clearly $-P \leq i \leq\lfloor P C R \times P\rfloor \leq$ $P$. The transition matrix is given by

$$
\mathbf{P}=\left(\begin{array}{lllllllll}
\sum_{i=-P}^{0} \mathbf{C}_{i} & \mathbf{C}_{1} & \ldots & \mathbf{C}_{P} & \mathbf{0} & \ldots & \mathbf{0} & \mathbf{0} & \ldots \\
\sum_{i=-P}^{-1} \mathbf{C}_{i} & \mathbf{C}_{0} & \ldots & \mathbf{C}_{P-1} & \mathbf{C}_{P} & \ldots & \mathbf{0} & \mathbf{0} & \ldots \\
\vdots & \vdots & \ddots & \vdots & \vdots & \ddots & \vdots & \vdots & \ddots \\
\mathbf{C}_{-P} & \mathbf{C}_{-P+1} & \ldots & \mathbf{C}_{0} & \mathbf{C}_{1} & \ldots & \mathbf{C}_{P-1} & \mathbf{C}_{P} & \ldots \\
\vdots & \vdots & \ddots & \vdots & \vdots & \ddots & \vdots & \vdots & \ddots
\end{array}\right)
$$

Remark that $\mathbf{P}$ is finite, but for simplicity reasons the boundary matrices are ommitted. By grouping the matrices $\mathbf{C}_{\mathbf{i}}$ in the appropriate way, a Quasi-BirthDeath (QBD) process is obtained, which can be solved by a classical algorithm e.g. the folding algorithm ([17]) or the logarithmic reduction algorithm ([10]). In both algorithms a rate matrix $\mathbf{R}$ is needed, of which the calculation can be improved using the specific structure of $\mathbf{P}$. The method is an extension of the matrix geometric algorithm introduced in [15] and can be found in [16].

\subsection{The Buffer Occupation in case $2 \tau>P$ and $p<<P$}

\section{(a) Evolution of the Process}

Now we consider the case where $2 \tau>P$, but with the additional assumption that the ON period of the CBR/VBR traffic is small with respect to the observation period. The interaction between the ABR-SES and the switch is illustrated in Figure 3. By choosing $2 \tau$ a multiple of $P$, say $2 \tau=L \times P$, we notice that the ACR during each observation period remains the same.

The basic idea is to use $L+1$ buffers for the ABR cells in stead of one. During the time intervals of the form $](2 \tau+P) n+(i-1) P,(2 \tau+P) n+i P[$ we will use the $i$ th buffer i.e. ABR cells that arrive during this interval will line up 


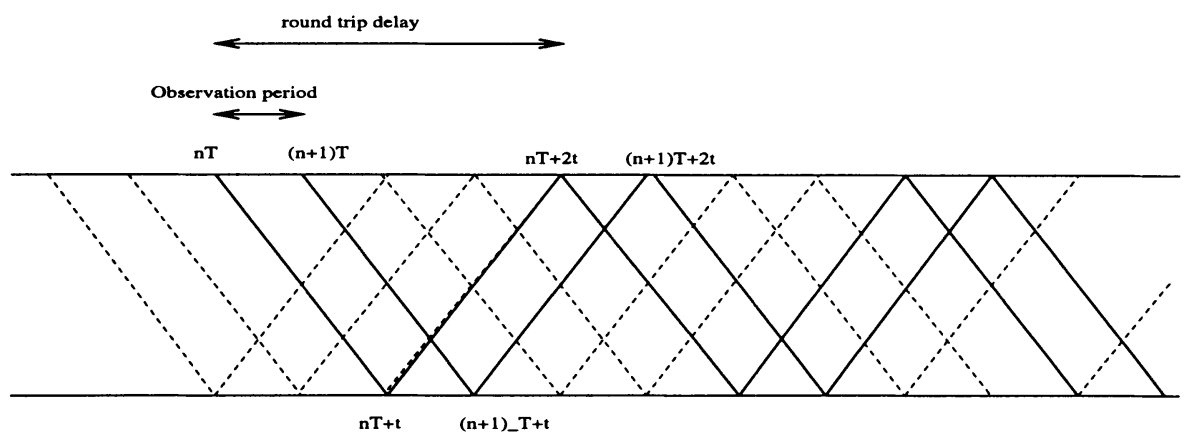

Figure 2 The interaction between Switch and ABR-SES, $2 \tau>P$

in the $i$ th queue and only cells of the $i$ th queue will depart during such an interval. Notice that the behavior of these $L+1$ buffer is somewhat different compared to the single buffer. Still given that each buffer contains at least one element it behaves exactly the same i.e. when a cell arrives or departs for the global queue so does one cell in the system with $L+1$ buffers. This allows us to believe that the tail of both distributions are very similar and it can be used when considering the cell loss ratio.

To solve the system with $L+1$ buffers we start by noticing that these $L+1$ buffers all have an identical distribution and given that $p \ll P$ they may be considered as independent. Thus once we have this unknown distribution the results are obtained by a simple convolution. This distribution is found by observing the system on the time instances $n(2 \tau+P)$ by means of the following vector $\bar{S}\left(p_{c}(n), p_{a}(n), r(n)\right)=\bar{S}(n)$, where $p_{c}(n)$ resp. $p_{a}(n)$ is the state of the D-BMAP used for modeling the CBR/VBR resp. ABR traffic at time $n(P+2 \tau)$ and $r(n)$ is the ACR for the time interval $] n(2 \tau+P), n 2 \tau+(n+1) P[$. The transition from $\bar{S}(n)=\left(i_{0}, j_{0}, \mu_{0}\right)$ to $\bar{S}(n+1)$ is similar to 3.1 .

\section{(b) Buffer Analysis}

The method used to solve the system introduced in section 3.2.1 is very similar to the one in section 3.1.2 and we can use the same technique. Since the dimensions of the matrices $\mathbf{C}_{\mathbf{i}}$ are reduced by a factor $v$, with $v$ the number of discrete values used for the ACR, the computational effort now is smaller.

\section{NUMERICAL EXAMPLES}

\subsection{Impact of the Burstiness and Observation Period Length on the Buffer Occupation}

In what follows we investigate the impact the burstiness of the CBR/VBR traffic and the duration of the observation period $P$ have on the ABR buffer occupation in the switch. In all the examples, by buffer occupation is meant 
the number of places in the buffer needed to guarantee a CLR $\leq 10^{-9}$. Example 1 : Consider the following system. The CBR/VBR traffic is modeled as a single ON/OFF source in order to better control its bursty nature. Let the ON period have a geometrically distributed duration with mean $p$ and let the OFF period have the same distribution with mean $q=1.25 \times p$. While in the ON state, the CBR/VBR traffic source generates a cell in each slot. Hence, the CBR/VBR traffic generates a load of 0.44 . Furthermore, we consider a greedy $\mathrm{ABR}$ traffic source with parameters $\mathrm{TCR}=0.9, \mathrm{PCR}=0.9$ and $\mathrm{MCR}=0.3$. The range of discrete values of the ACR is [ 0.30 .450 .60 .750 .9$]$. We let the round trip delay be $\tau=1$. We investigate the buffer occupation for variable mean ON period duration $p$ and variable observation period length $P$.

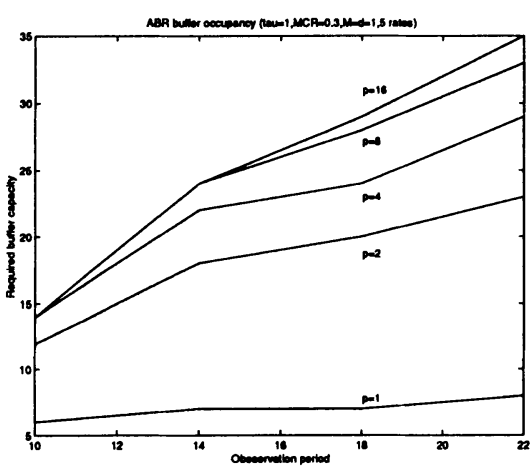

Figure 3 The buffer occupation as function of $P$ and $p, p \leq 16$

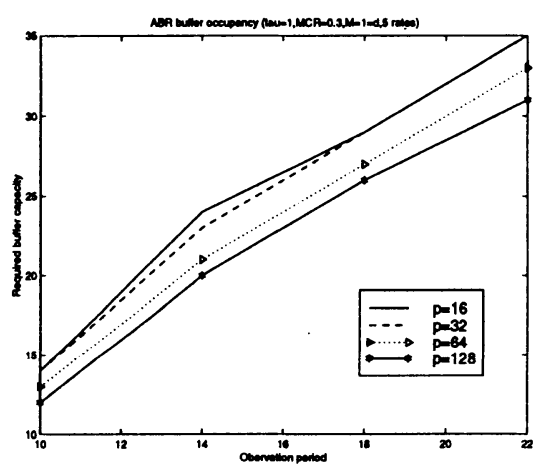

Figure 4 The buffer occupation as function of $P$ and $p, p \geq 16$

Figure 4 shows the impacts for mean ON periods smaller than 16 when $P=20$. In this case the buffer occupation grows with the length of the ON period. As soon as the mean ON duration $p$ is larger than 16 as shown in Figure 5, the buffer occupation as function of $p$ shows a decreasing tendency. Note that 16 is about the same magnitude as the length of the observation period $P$. This observation is confirmed in Figure 6, where the buffer occupation is depicted as a function of the mean ON period duration $p$ for different values of the observation period length $P=20,60,120$. From this figure we conclude that the buffer occupation reaches a maximum for values of $p$ around or just before the length of the observation period $P$. In Figure 7, we show how the buffer occupation reaches slowly a stable value (and slightly decreasing thereafter) when the observation period duration $P$ is increased. Moreover, we see that the convergence is slower for higher burstiness of the CBR/VBR traffic source. 


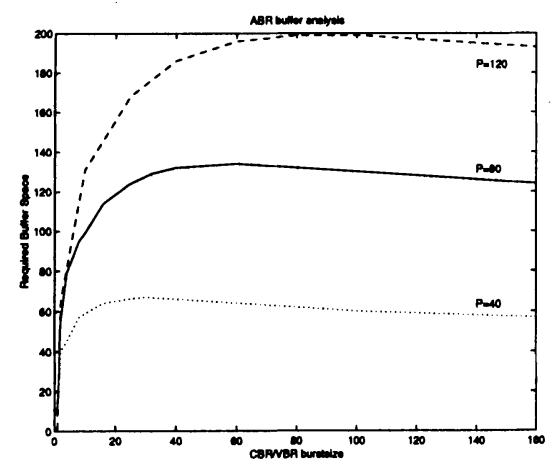

Figure 5 Maximal Buffer Occupation for $P=40,80$ and 120 .

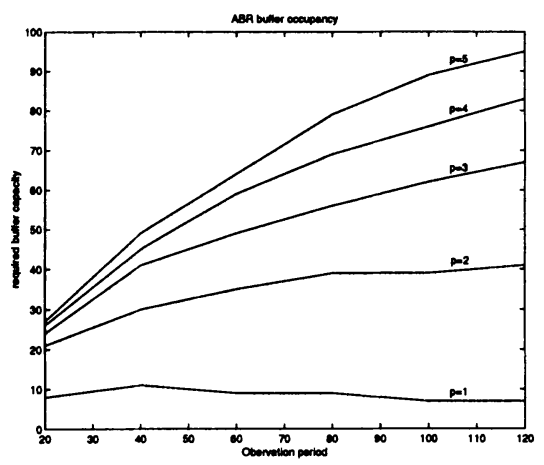

Figure 6 The Buffer Occupancy for High Values of $P$

\subsection{Impact of the Burstiness and Round-Trip Delay on the Buffer Occupation}

Let us now investigate the impact of the round-trip-delay $\tau$, and the burstiness of CBR/VBR traffic on the ABR buffer occupation.

Example 2 : $2 \tau \leq P$ : We consider a system similar to the one in Example 1 . The observation period length is chosen to be constant and equal to $P=20$. The round trip delay $\tau$ varies between 1 and $P / 2$. Hence we apply the analysis of Section 3.1. Figure 8 illustrates the fact that from a certain value of the

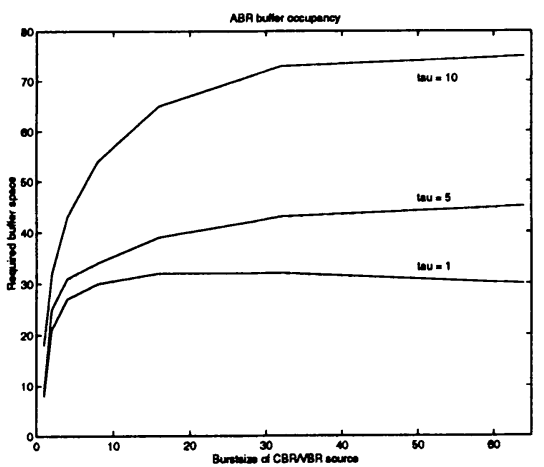

Figure 7 The Buffer Occupation as function of $\tau$

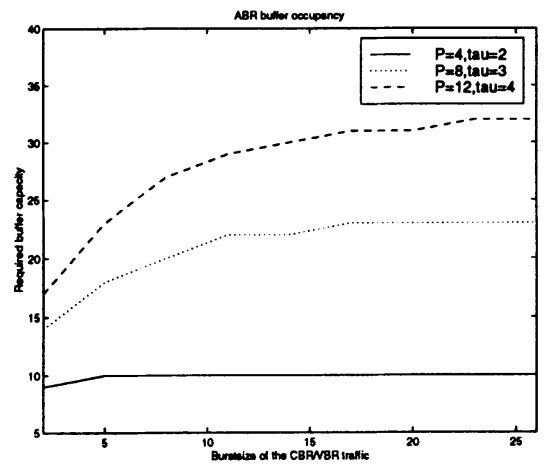

Figure 8 The Buffer Occupation as function of $\tau$ and $P$

burstsize of the CBR/VBR traffic, the buffer occupation remains constant (or even slightly decreases). From the figure it follows that this point is situated just before $20+2 \times \tau$. This rule can be generalized as follows. In a system with observation period $P$ and round trip delay $\tau$, the buffer occupation remains constant for mean ON period values $p \geq 2 \times \tau+P$. This property is illustrated in Figure 9, where the buffer occupation is shown as a function of the burstsize, 
for variable $P$ and $\tau$.

Example 3 : $2 \tau>P$ : Consider again the system described in Example 1 and let $\tau$ vary between 0 and 120, incrementing in steps of 20 . Furthermore, we let $P=20$ and $P=40$ and we let the on period of the CBR/VBR traffic be $p=2$ and $p=4$. Figure 10 shows the that increasing $\tau$ leads to large buffers.

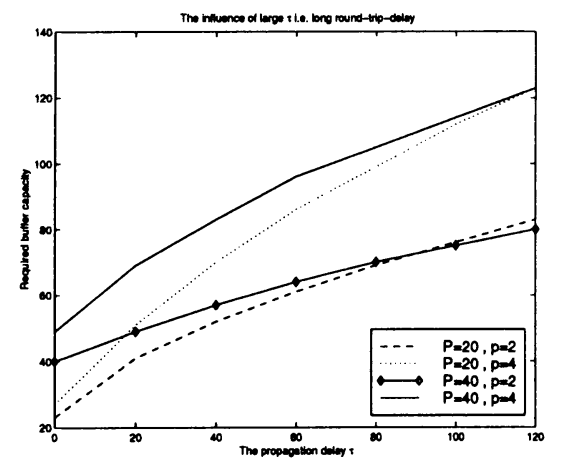

Figure 9 The Buffer Occupancy as function of $\tau$ for different values of $P$

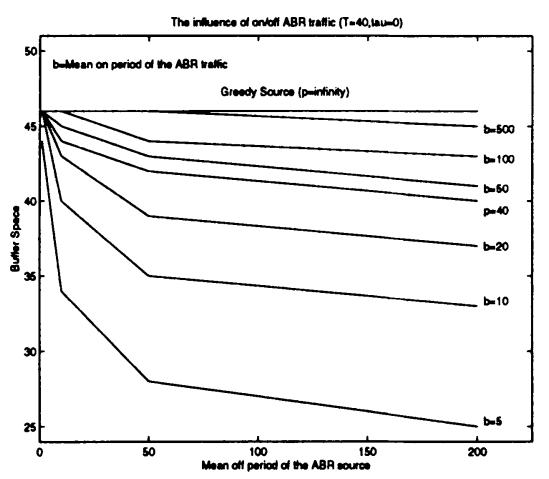

Figure 10 Modeling ABR traffic as bursty or as greedy

Even for high values of $\tau$ with respect to $p$, the required buffer size is still increasing for growing $\tau$. This is in contrast with the corresponding results for $P$ (see Figure 7). An important conclusion to be drawn from Figure 10 is that the value of $P$ becomes irrelevant for values of $\tau$ much larger than $P$.

\subsection{Modeling ABR as Greedy or Bursty Sources}

To conclude this set of numerical result we turn our attention to the modeling aspect of the ABR traffic. It is clear that incorporating burstiness of the ABR traffic into the model increases its complexity. In the following example we investigate when we may ignore the bursty character of the ABR traffic.

Example 4: Consider a system consisting of the following components. A CBR/VBR traffic source which has an ON/OFF behavior with mean on period $p=50$, mean on period $q=62.5$ and probability of generating a cell when $\mathrm{ON}$ equal to 1 , resulting in a load of 0.44 . The ABR traffic has parameters $\mathrm{TCR}=0.9, \mathrm{PCR}=0.9$ and $\mathrm{MCR}=0.15$. The round trip delay is assumed to be $\tau=0$, while $P=40$. Now let the ABR have increasing mean ON period, where the value infinity corresponds with a greedy source. Multiple numerical results have shown (see e.g. Figure 11) that the needed buffer capacity is approximated very well by a greedy source as soon as the mean ON period of the ABR traffic reaches the value $2 \times \tau+P$, even for small ABR loads. 


\section{CONCLUSIONS}

This paper presents an analytical evaluation of the buffer occupancy of ABR traffic in a switch when sharing the outgoing link with CBR/VBR traffic and using an Explicite Rate congestion control scheme. From the numerical examples we may draw the following conclusions and derive some engineering rules.

- The burstiness of the CBR/VBR traffic has a strong influence on the required buffer capacity. As long as $P+2 \tau$ is longer than the mean burstsize, the ABR buffer occupation increases with increasing CBR/VBR burstiness. If the mean burstsize is larger than $2 \tau+P$, then the burstiness has no real impact on the used buffer space. It follows, that in order to find the maximum value of the buffer occupation it is sufficient to consider CBR/VBR traffic with mean burst duration of the order of $2 \tau+P$.

- When selecting the length of the observation period $P$, one may take into account the following observation. The larger $P$ the more buffer space is needed to guarantee a low CLR, thus we can gain buffer space by reducing the length of the observation period this at the price of increasing the number of RMcells i.e. the network overhead. But no relevant gains in buffer occupation can be made by changing its length $P$ as long as is stays well above the mean length of the burstsize of the CBR/VBR traffic.

- The longer the distance between the ABR-SES and the switch, the more buffer space is needed even when the mean burst size is much smaller than the delays considered.

- In networks with large-round trip delays relative to the observation period, no gain can be made by adapting the length of the observation period, but such that it remains small compared to the round-trip delay.

- When modeling the Explicit Rate congestion control scheme, on may replace the bursty ABR traffic by the more simple model of greedy sources in case the mean ON periods of the on/off ABR traffic are larger than the sum of the length of the observation period $P$ and the round-trip-delay $2 \tau$, even if the load of the on/off sources is well below the value of 0.1 .

\section{Acknowledgements}

This work was supported in part by the Commission of the European Union, under project ACTS AC094 "EXPERT". The first author was also supported by Vlaams Actieprogramma Informatietechnologie under project ITA/950214 "Design and Control of Broadband Networks for Multimedia Applications".

\section{REFERENCES}

[1] ATM Forum, ATM Forum Traffic Management Specification, Version 4.0, April 1996. 
[2] A. W. Barnhart, Explicit Rate Performance Evaluation, ATM Forum document AF-TM-94-0983R1.

[3] C. Blondia, O. Casals, Performance Analysis of Statistical Multiplexing of VBR Sources, IEEE Infocom'92, Florence (Italy), 1992.

[4] C. Blondia and O. Casals, Statistical multiplexing of VBR sources : A matrix-analytic approach, Performance Evaluation, 16 (1992) 5-20.

[5] C. Blondia and O. Casals, Analysis of Explicit Rate Congestion Control in ATM Networks, Proceedings Australian Telecommunications Networks and Applications Conference (ATNAC '96), December 1996, Melbourne, Australia, 1996

[6] C. Blondia and O. Casals, Throughput analysis of the explicit rate congestion control mechanism, Proceedings 10th ITC Specialist Seminar, Lund, Sweden, 1996

[7] R. Jain, S. Kalyanaramam and R. Viswanathan, The EPRCA+ scheme, ATM Forum document 94-1173

[8] R. Jain, A sample switch algorithm, ATM Forum document 95-0178R1

[9] R. Jain, S. Kalyanaramam, R. Goyal, S. Fahmy and R. Viswanathan, ERICA switch algorithm: A complete description, ATM Forum document AF-TM-96-1172.

[10] G. Latouche and V. Ramaswami, A logarithmic reduction algorithm for Quasi-Birth-Death processes, J. of Appl. Prob., 30 (1993) 650-674

[11] M.F. Neuts, Matrix-Geometric Solutions in Stochastic Models, The John Hopkins University Press, Baltimore, 1981

[12] H. Ohsaki, M. Murata, H. Suzuki, C. Ikeda and H. Miyahara, RateBased Congestion Control for ATM Networks, ACM SIGCOM Computer Communication Review, (1995) 60-72

[13] M. Ritter, Analysis of Feedback-Oriented Congestion Control Mechanisms for ABR Services, ITC Specialist Seminar on Control in Communications, Lund (Sweden), 1996.

[14] M. Ritter, Network Buffer Requirements of the Rate-based Control mechanism for ABR Services, IEEE INFOCOM'96 proceedings, San Francisco, (1996) $1190-1197$

[15] K. Wuyts and R. K. Boel, A matrix geometric algorithm for finite buffer systems with B-ISDN applications, ITC Specialist Seminar on Control in Communications, Lund (Sweden), 1996.

[16] K. Wuyts and B. Van Houdt, Matrix geometric analysis of discrete time queues with batch arrivals and batch departures, in preparation

[17] Jingdong Ye and San-qi Li, Folding Algorithm, A computational method for finite $Q D B$ processes with level-dependent transitions, IEEE Trans on Comm., 42(2/3/4) (1994) 625-639

[18] N. Yin and M.G. Hluchyj, On Closed-Loop Rate Control for ATM Cell Relay Networks, IEEE INFOCOM'94, Toronto. 